\title{
The usefulness of periostin determination in gynecology and obstetrics
}

\author{
Christopher Kobierzycki1 ${ }^{10}$, Krzysztof J. Latkowski², Piotr Dziegiel ${ }^{1,3}$ \\ ${ }^{1}$ Division of Histology and Embryology, Department of Human Morphology and Embryology, \\ Wroclaw Medical University, Wroclaw, Poland \\ 2 I Department of Obstetrics and Gynecology, Wroclaw Medical University, Wroclaw, Poland \\ ${ }^{3}$ Department of Physiotherapy, Higher School of Physical Education, Wroclaw, Poland
}

\begin{abstract}
Periostin (POSTN) is a multifunctional glycoprotein that belongs to the group of extracellular matrix (ECM) proteins. Due to the molecular structure, cellular interactions, tissue locations as well functions of POSTN, we realize that its pivotal role is organization and regulation of ECM microenvironment. In available databases there is a lack of data summarizing current knowledge about POSTN expression in the field of gynecology and obstetrics. We conducted a search in PubMed of the National Library of Medicine and Google Scholar. Databases were extensively searched for all original and review articles/book chapters published in English until December 2019 and related to periostin expression. All relevant articles were reviewed and presented as appropriate.

In the field of POSTN expression there is only one paper evaluating its involvement in cervical cancer cell metabolism and only two studies analyzing its myometrial commitment: maintenance during pregnancy and induction of parturition in physiology as well control of fibroids biology in pathology. Much more attention has been devoted to the expression of described protein in the endometriosis, and above all in ovarian cancer. Finally, a few studies carried out among pregnant women were presented.

In this review study we presented current knowledge about periostin expression in the field of gynecology and obstetrics. Many achieved results are interesting and further studies are needed to verify some hypotheses. Structure, signaling pathways as well many functions of periostin are well-described. However, as it was clearly shown there is a lot of unknown issues which are waiting to be explored.
\end{abstract}

Key words: periostin; POSTN; cancer; neoplasm; endometriosis; myoma; leiomyoma; fibroid; gestation; pregnancy; miscarriage

Ginekologia Polska 2020; 91, 6: 346-351

\section{INTRODUCTION}

Periostin (POSTN) also called osteoblast-specific factor 2 (OSF-2) is a multifunctional glycoprotein that belongs to the group of extracellular matrix (ECM) proteins. Physiological POSTN expression was disclosed in wide variety of normal fetal and adult tissue; i.a. embryonic periosteum, periodontal ligament, placenta, cardiac valves, adrenal glands, lung, thyroid, stomach, colon, vagina, ovary, testis, prostate, and breast. It has been shown to be an important regulator of bone and tooth formation and maintenance, and cardiac development and healing [1, 2]. Additionally, it was also found that POSTN is expressed in tissues under stress conditions, such as pressure or volume overload in heart, skeletal muscle after injury or cellular response to hypoxia. Moreover, POSTN overexpression has been reported for some pathological states, i.e. chronic sinusitis, allergic airway inflammation as well in many cancer types [1-3].

The human POSTN is encoded by gene located on chromosome 13q13.3, and has two isoforms (779 and 836 -amino acid protein with a molecular weight of $87 \mathrm{kDa}$ and $93.3 \mathrm{kDa}$, respectively) identified through studies on human placenta and osteosarcoma cDNA libraries [2]. POSTN has an N-terminal signal peptide, a cysteine-rich region, four internal homology domains and a carboxyl terminal region. There is a typical signal sequence at the $\mathrm{N}$-terminus suggesting that it has the potential to be a secreted protein. The C-terminus is hydrophilic and can undergo alternative splicing at the transcriptional level to form splice variants or 
isomers of POSTN. It has eight types of homologous isoforms in human tissues that are associated with the occurrence of specific tumors [1, 3].

POSTN by interaction with the integrins $\left(\alpha_{v} \beta_{3}, \alpha_{v} \beta_{5}\right.$, and $\alpha_{6} \beta_{4}$ ) activates the Ilk-PI3K/Akt and focal adhesion kinase (FAK)-mediated signaling pathways. It is responsible for ECM remodeling, as it controls adhesion processes and biomechanical properties of connective tissue (i.a. regulation of collagen fibriollogenesis) $[4,5]$. It creates an environment for angiogenesis and lymphangiogenesis, independent proliferation, avoidance of apoptosis and the ability for cells to re-enter the cell cycle, which in pathology leads to promotion of cancer cell survival, invasion and metastases, mostly on basis of epithelial-mesenchymal transition (EMT) phenomenon $[5,6]$.

Due to the molecular structure, cellular interactions, tissue locations as well functions of POSTN we realize that its pivotal role is organization and regulation of ECM microenvironment. In available databases there is lack of data summarizing current knowledge about POSTN expression in the field of gynecology and obstetrics.

\section{Methodology of Data Obtaining and Analysis}

We conducted a search in PubMed of the National Library of Medicine and Google Scholar. Databases were extensively searched for all original and review articles/book chapters published in English until December 2019 and related to periostin expression using the following keywords (one or in combinations): periostin, POSTN, gynecology, cancer, neoplasm, endometriosis, myoma, leiomyoma, fibroid, obstetrics, gestation, pregnancy, miscarriage. Moreover, additional articles from the reference sections of the reviewed articles were searched. Overall, all relevant articles were reviewed and presented as appropriate.

\section{Cervix}

In the field of cervical expression of POSTN there is only one paper evaluating its involvement in cancer cell metabolism. Han et al. investigated human cervical cancer samples as well commercial cell lines (HeLa and $\mathrm{SiHa}$ ). They disclosed overexpression of POSTN in studied cases. Moreover, they observed that POSTN knockdown in tested cell lines significantly decreased cell viability, migration and invasion, and reduced EMT. Furthermore, POSTN knockdown suppressed the activation of Akt/mTOR signaling pathway. In well-designed experiment Han et al. [7] demonstrated that of MALAT-1/microRNA-202-3/POSTN axis regulates cell viability, migration and invasion as well EMT of cancer cells via Akt/mTOR signaling pathway. Further research would be greatly beneficial, especially due to unknown prognostic and predictive value of this protein in cervical cancer.

\section{Endometrium}

The endometrium is composed by two types of cells i.e. endometrial stromal cells (ESC) and endometrial epithelial cells (EEC) which both may have eutopic (physiological) and ectopic (pathological) localization. First study on POSTN expression in eutopic endometrium was conducted by Hiroi et al. [8] in 2008. A significant increase in POSTN expression was observed during mid-proliferative and early secretory phases while during late proliferative, mid-secretory and late-secretory phases were decreased. Observations strongly correlated with estrogen and progesterone supplementation during the study. Moreover, they disclosed increased immunohistochemical (IHC) POSTN expression in ESC during early proliferative, mid-proliferative and early secretory phases as well in EEC in late secretory phase. The authors clearly showed that POSTN expression is controlled by ovarian steroid hormones, which may have strong impact on physiological pregnancy and pathological processes e.g. endometriosis.

Endometriosis is a chronic inflammatory disease caused by ectopic endometrium manifesting itself by recurrent pain and infertility in women of reproductive age. Repeated tissue injury, repair and subsequent fibrosis are its crucial elements. Microscopic image is characteristic and well-described, however, due to lack of known etiology as well signaling pathways, further studies are needed. Therefore, Shen et al. [9] investigated IHC POSTN expression in eutopic and ectopic endometrium of women diagnosed with endometriosis. They observed cyclic variation of eutopic stromal IHC POSTN expression; stronger in the proliferative than in the secretory, similarly to Hiroi's observations. Moreover, Shen et al. found the strongest POSTN expression in ectopic endometrium, which suggests involvement of POSTN in the pathophysiology of endometriosis. In $2015 \mathrm{Xu}$ et al. [10] studied mRNA and protein expression of POSTN in ectopic ESC. They disclosed that POSTN enhanced ESC migration, invasion as well adhesion due to the Ilk/Akt signaling pathway. Moreover, they speculated that due to the known way of action, POSTN may be a new therapeutic target in endometriosis. Based on the above data, Zheng et al. [11] decided to verify POSTN expression in EEC and check its influence on EMT. They received results concordant with those by Xu et al., i.e. POSTN enhanced invasion and migration abilities of EEC as well as, facilitated the EMT through Ilk-Akt signaling pathway. Further studies conducted in 2018 by Logan et al. [12], investigated molecular pattern of EEC and ESC of eutopic endometrium women with endometriosis. They performed fluorescence-activated cell sorting for paired sibling RNA sequencing and miRNA microarray and disclosed 151 and 215 differently expressed genes as well 9 and 16 differently expressed miRNAs in ESC and EEC, respectively. POSTN was found to be downregulated in ESC. 
Results of this study may be crucial in perspective of eutopic endometrium biology in patients affected by endometriosis and their pregnancy expectations.

Other studies on POSTN expression in endometriosis are focused on identification therapeutic approach. Ganieva et al. determined whether transcription factor 21 (TCF21) is involved in the development of endometriosis as an upstream regulatory gene of POSTN [13]. They separately verified expression of POSTN and TCF21 in different types of endometriosis. They disclosed that expression of POSTN and TCF21 was absent in normal endometrium of women without endometriosis, weakly positive in eutopic endometrium of women with endometriosis, moderately positive in ovarian endometriosis, and strongly positive in deep infiltrating endometriosis. Additionally, usage of siRNA against human TCF21 suppressed POSTN expression, as well transfection of TCF21 plasmid vector into stromal cells of women without endometriosis, which natively expressed neither POSTN nor TCF21, resulted in presence of their expression. In view of the above information, Ganieva et al. suggest that TCF21 may be promising therapeutic target in endometriosis.

\section{Myometrium}

The myometrial expression of POSTN is described only in two studies. Liu et al. [14] analyzed molecular mechanism responsible for balance between maintenance of pregnancy and induction of parturition. They compared contracted lower uterine segment (LUS) with relaxed uterine fundal myometrium (FUN) to assess transcriptome by RNA-sequencing, then RT-PCR and immunoblotting to validate sequencing results. Finally, cell contraction/adhesion assays and gene microarrays were used to study the cellular functions of identified genes. They disclosed higher levels of HoxA13, PTGIS, and POSTN in LUS at term prior to labor. The authors stated that regionalization of myometrial function may be mediated by HoxA13. Liu et al. [15] disclosed myometrial function of POSTN in physiology, however it was suspected to have its role in pathology. Jamaluddin et al. performed proteomic profiling of uterine fibroids to analyze ECM protein expression pattern. Using genetic sequencing and isobaric tagged-based quantitative mass spectrometry (iTRAQ) they analyzed samples in two main groups: MED1 2 positive and negative mutation (one of the most common anomalies in fibroids). They disclosed sets of down and upregulated proteins, however regardless MED12 status POSTN was significantly upregulated. Performed western blotting (WB) and IHC analyses confirmed proteomic observations. They finally concluded that increased POSTN is a hallmark of uterine fibroids regardless MED12 status and further studies on up-regulated ECM proteins should be performed.

\section{Ovary}

Polycystic ovary syndrome (PCOS) is often diagnosed a metabolic disease associated with hormonal imbalance. There is growing evidence for links between POSTN expression and ovarian function. Chen et al. [16] evaluated by ELISA POSTN levels and compared it with parameters of triglyceride metabolism, chronic inflammation, and insulin resistance in PCOS patients. Serum POSTN levels in PCOS patients were almost 10 times higher than in controls. They discovered also that POSTN positively correlates with body mass index (BMI), uric acid, homeostasis model assessment of insulin resistance (HOMA-IR), high-sensitive $C$ reactive protein (hs-CRP), and negatively with insulin sensitivity index (ISI). Chen et al. finally concluded that elevated levels of POSTN are associated with PCOS. In perspective of incidence of PCOS, presented results and lack of studies, further analysis is essential.

Tumor-specific molecular modifications have their place in the management of oncologic patients. POSTN is one of the glycoproteins identified to have it role in ovarian cancer (OC) [17]. One of the first studies showing overexpression of POSTN in OC was presented Gillan et al. in 2002. They concluded that POSTN can increase the motility of the cancer cells and their adhesion to the peritoneum via integrins $a_{v} \beta_{3}$ and $\alpha_{v} \beta_{5}$. It results in detection of POSTN in the ascites of OC patients [18]. In the following studies POSTN properties were evaluated. Zhu et al. tested POSTN expression by CDNA microarray, northern blotting and IHC analyses [19]. Subsequently, using retroviral transfection, supported by WB and ELISA confirmation, they achieved POSTN expression in OC cells lines: OVCAR-3 and OV2008. The clinical effects of exogenous POSTN expression were assessed in orthotopic mouse models. Zhu et al. [19] found that POSTN overexpression did not change cell growth rates in vitro; however, it significantly promoted intraperitoneal tumor metastatic growth in immunodeficient mice, which was associated with increased tumor angiogenesis and decreased tumor cell apoptosis. It stays in line with the study by Choi et al. [20], who analyzed association between POSTN expression and lysophosphatidic acid (LPA), bioactive lipid crucial for the initiation and progression of OC. Treatment of SKOV-3 and OVCAR-3 cell lines with LPA induced expression of POSTN. Additionally, induced POSTN expression was abrogated by silencing of the LPA receptor expression using shRNA lentivirus. Recombinant POSTN stimulated adhesion and invasion of SKOV-3 cells what suggests that LPA may be associated with POSTN expression in the OC.

Subsequently, Abbot et al. performed glycotranscriptome comparative analysis of tissue and serum patients with OC to identify new specific markers. Selected transcripts of restricted glycosyltransferases were verified by lectins, 
which showed tumor-specific glycosylation changes. The authors stated that regarding tested glycoprotein markers, thrombospondin and POSTN were overexpressed in OC and they can be potentially used to distinguish OC patient serum from normal serum [21]. Furthermore, proteomic study conducted by Tian et al. was aimed on identification glycoproteins useful in proper differentiation of histological subtypes of OC. The authors utilizing ITRAQ and WB disclosed that POSTN is overexpressed in most subtypes of OC and is unsuitable for differentiation. However, Tian et al. [22] simultaneously pointed its potential involvement in an early stages of OC formation, what may also constitute promising therapeutic target.

There are hypotheses that POSTN cleavage by blocking antibody or induced transcriptional gene silencing may constitute a highly effective method of treatment. Zhu et al. [23] developed neutralizing monoclonal antibody against POSTN called MZ-1. They performed their study on OC mice model derived from cell line A2780 to verify impact of MZ-1 on tumor growth and metastasis. The Authors believed that observed inhibited peritoneal metastasis after treatment with MZ-1 was result of specific inhibition of anchorage-independent growth and survival of POSTN expressing cells as well neutralizing effect of POSTN induced cancer cell migration and invasion. Moreover, in vivo administration of MZ-1 resulted in reduction in the number of metastasis.

In the subsequent studies prognostic and predictive value of POSTN was verified. Karlan et al. [24] performed RNA expression microarrays and Rosetta Similarity Search Tool to define sets of genes with potential relevance in $\mathrm{OC}$, which finally were identified by array comparative genomic hybridization. They pointed out that high-grade serous OC patients with POSTN/TGFBI expression have significantly shorter overall survival (OS) being potential prognostic and therapeutic target. Ryner et al. [25] performed gene expression profiling on a discovery set of OCs with clinically well-defined response to chemotherapy as well as on an independent validation dataset of $O C$ patients from the chemo treatment arm of the ICON7 trial. They defined "reactive stroma" gene signature that is specifically associated with primary chemo resistant tumors. IHC and RNA in situ hybridization analyses confirmed POSTN, LOX, and FAP genes to be associated with the clinical chemo resistance. Moreover, treatment with recombinant POSTN promoted ES-2 cell to carboplatin and paclitaxel treatment in vitro. Finally, they demonstrated that a high POSTN expression level predicts shorter progression-free survival (PFS) following first-line chemotherapy. In perspective of above observations and own study, Lister et al. found that promoter-directed small antisense non-coding RNAs can induce transcriptional gene silencing of POSTN what may result in a loss of cellular motility. Moreover, they observed that cell motility and possibly metastasis can be controlled by transcriptional and epigenetic regulation of POSTN [6].

In 2015 Tan et al. [26] presented transcriptomics database of human OC, referred to as CSIOVDB which comprises 3,431 microarray samples from 48 cohorts of private, in-house and public human $O C$ datasets. They observed that elevated POSTN expression was characteristic for serous OC vs other histotypes, stage II-IV vs I, grade $2-3$ vs 1 as well tumor resistant or refractory to first line chemotherapy vs sensitive ones. Moreover, Tan et al. [26] pointed that POSTN levels were significantly associated with OS, DFS as well showed that it was an independent predictor of PFS. Similarly, Sung et al. [27] analyzed microarray datasets and therefore performed in vitro study with A2780 cell line. They observed that POSTN treatment induced cisplatin resistance and activate Akt pathway, while its inhibition by selective inhibitor, MK-2206 abolished POSTN-induced Akt activation and cisplatin resistance in vitro. Overall, they showed that overexpression of stromal POSTN has independent poor prognostic value (OS and DFS) and is associated with higher percentage of platinum resistance. In 2018 Tang et al. [28] disclosed high POSTN levels in ascites OC patients (the same as Gillan et al. [18]) which correlated with level of CD163 tumor-associated macrophages. The high POSTN level and macrophage infiltration were inversely associated with relapse-free survival for $O C$ patients. Additionally, with techniques described by Zhu and Lister, siRNA of POSTN and POSTN neutralizing antibody treatment showed that OC cell derived POSTN promoted the recruitment of macrophages and modulated their cytokine secretion profile [28].

In most up-to date studies, in 2019, Lu et al. [29] generated biotinylated form of human scFv antibody that targets the bisected $\mathrm{N}$-glycans. They validated results in in vitro and in vivo studies indicating that obtained antibody may be useful for development diagnostic and therapeutic approaches for cancers expressing POSTN. Moreover, Sterzyńska et al. [30] investigated POSTN mRNA by RT-PCR and protein expression in cell lysates and cell culture medium by WB as well in cell lines by IF regarding development of chemotherapeutic resistance. They disclosed increased expression of POSTN in drug-resistant cells and stated that POSTN expression might be associated with the development of doxorubicin and methotrexate resistance in the primary serous OC cell line. Finally, in 2020 Kujawa et al. [31] analyzed expression of POSTN and fibronectin. They disclosed that higher expressions of both proteins were associated with shorter OS and demonstrated that combined score of fibronectins and POSTN was an independent prognostic factor for OS. What is new, Kujawa et al. [31] stated that elevated expression of fibronectin and POSTN was more common in fallopian cancers than in OC. 


\section{Pregnancy}

It has been shown that molecular processes occurring in the cancer cell metastasis are like those observed during human implantation. In view of above, ECM proteins responsible for cell adhesion allowing cancer cell maintenance may also have an impact on implantation. Therefore, Morelli et al. [32] analyzed tissue and serum expression of POSTN during pregnancy. They analyzed mRNA and protein expression in decidual and trophoblastic tissue as well serum protein level in between group of pregnant with spontaneous pregnancy loss and planned pregnancy termination. In all analyzed aspects POSTN expression was elevated in spontaneous pregnancy loss. The Authors speculated that POSTN may be a serum marker of good endometrial receptivity, embryo quality and predictive for pregnancy evolution. In the other study Freis et al. [33] tested by ELISA levels of POSTN in patients who conceived by IVF/ICSI and ovarian stimulation and with known first trimester outcome. They disclosed that increased levels of POSTN where observed in early pregnancy in patients with following miscarriage in contrast to patients with ongoing pregnancy who demonstrated decrease in POSTN levels. They hypothesized that POSTN is potential promising marker for assessment of pregnancy outcome [33]. Both presented studies have consistent results, however, their serious limitations are small study groups which should be expanded to strengthen conclusions.

In an interesting paper, Song et al. [34] presented proteomic analysis of neonatal umbilical cord serum. They disclosed differences in protein expression between fetal gender. They concluded that POSTN expression is consistent with the marked rate of growth and development of the fetus. Moreover, they identified 61 neonatal specific proteins that were absent in the adult plasma proteome, i.a. POSTN. It seems contrary to the results of many other research teams, who demonstrated presence of POSTN in adults' sera. Song et al. [34] analyzed arterial umbilical blood obtained during vaginal term delivery. Placental ablation may potentially influence the POSTN level so at least venous umbilical blood should be checked. Optionally, blood from cordocentesis would be particularly useful for comparative study. The results should also be analyzed bearing in mind the study by Sasaki et al. [35] in 2002 who speculated POSTN role in the pathogenesis of preeclampsia. They tested blood by novel sandwich chemiluminescence assay as well as placenta by using RT-PCR and in situ hybridization of preeclampsia and normotensive pregnant women. They found elevated serum POSTN levels and its increased expression in placental stromal cell. The authors hypothesized that serum POSTN might be a novel marker of preeclampsia in mechanism of release from the placenta due to disturbed adhesive interactions between cells or as an element of inflammatory process connected with activation of leukocytes and endothelial cells. The exact function of POSTN remains unclear, but the fact that it is an adhesion molecule in view of achieved results suggest novel mechanisms in preeclampsia.

Regarding known functions and interactions of POSTN there are some potential clinical implications which should be evaluated. Dobreva et al. [36] decided to investigate bone morphogenetic protein (BMP) target genes, putative amniotic membrane markers in mouse. There is evidence that deficiency in one of several components of the BMP signaling cascade in mice resulted in defective development of the early amnion. In comparative gene expression analysis of acknowledged target genes for BMP in different extraembryonic tissues, they disclosed by in situ hybridization POSTN mRNA enrichment in amnion throughout gestation. The Authors suggested usage of POSTN and protein AP-2 as a transcriptional signature for different mouse extraembryonic tissues. Until now, no human studies were conducted. Moreover, Ivancsó et al. [37] performed a study analyzing plasma levels of POSTN in pregnant women with asthma, as there is already strong evidence that POSTN is a useful biomarker in asthmatic patients. The authors stated that POSTN correlates with lung function in asthmatic pregnancy and it may play role in a normal gestation but should be handled with caution in pregnant women due to the possible influence of pregnancy on its plasma levels. Additionally, Świrska et al. [38] prepared very comprehensive review on selected cytokines and hormones of confirmed or possible role in pathogenesis of gestational diabetes mellitus. They cautioned about substantial facts, i.e. known relation between POSTN concentration and metabolic diseases as POSTN enhances c-Jun N-terminal kinase-dependent suppression of fatty acids oxidation in the liver and enhanced POSTN expression by higher glucose concentrations. Moreover, they showed many annotations in the literature which point to the possible involvement of POST in gestational diabetes mellitus. However, surprisingly and sadly in available databases there is lack of studies analyzing mentioned above possible relationship.

\section{CONCLUSIONS}

In this review study we presented current knowledge about periostin expression in the field of gynecology and obstetrics. Many achieved results are interesting and further studies are needed to verify some hypotheses. Structure, signaling pathways as well many functions of periostin are well-described. However, as it was clearly shown there is a lot of unknown issues which are waiting to be explored.

\section{Conflict of interest}

The authors declare that there are no conflicts of interest. 


\section{Acknowledgments}

The study performed as a part of the project entitled „Expression of periostin in invasive and preinvasive cervical lesions" and financed by grant of the Polish Society of Gynecologists and Obstetricians in Young Researcher Competition.

\section{REFERENCES}

1. Morra L, Moch H. Periostin expression and epithelial-mesenchymal transition in cancer: a review and an update. Virchows Archiv. 2011; 459(5): 465-475, doi: 10.1007/s00428-011-1151-5.

2. Horiuchi K, Amizuka N, Takeshita S, et al. Identification and Characterization of a Novel Protein, Periostin, with Restricted Expression to Periosteum and Periodontal Ligament and Increased Expression by Transforming Growth Factor $\beta$. Journal of Bone and Mineral Research. 1999; 14(7): 1239-1249, doi: 10.1359/jbmr.1999.14.7.1239.

3. Ye D, Shen Z, Qiu S, et al. Role and underlying mechanisms of the interstitial protein periostin in the diagnosis and treatment of malignant tumors (Review). Oncology Letters. 2017, doi: 10.3892/ol.2017.6866.

4. Ruan K, Bao S, Ouyang G. The multifaceted role of periostin in tumorigenesis. Cellular and Molecular Life Sciences. 2009; 66(14): 2219-2230, doi: 10.1007/s00018-009-0013-7.

5. Ratajczak-Wielgomas K, Dziegiel P. The role of periostin in neoplastic processes. Folia Histochemica et Cytobiologica. 2015; 53(2): 120-132, doi: 10.5603/fhc.a2015.0014.

6. Lister N, Clemson M, Morris K. RNA-directed epigenetic silencing of Periostin inhibits cell motility. Royal Society Open Science. 2015; 2(6): 140545, doi: 10.1098/rsos.140545.

7. Han X, Wang Q, Wang Y, et al. Long non-coding RNA metastasis-associated lung adenocarcinoma transcript 1/microRNA-202-3p/periostin axis modulates invasion and epithelial-mesenchymal transition in human cervical cancer. Journal of Cellular Physiology. 2019; 234(8): 14170-14180, doi: 10.1002/jcp.28113.

8. Hiroi $\mathrm{H}$, Momoeda $\mathrm{M}, \mathrm{Nakazawa} F$, et al. Expression and regulation of periostin/OSF-2 gene in rat uterus and human endometrium. Endocr J. 2008; 55(1): 183-189, doi: 10.1507/endocrj.k07-073, indexed in Pubmed: 18270434.

9. Shen L, Liu P, Zhang P, et al. Characterization of periostin expression in human endometrium and endometriotic lesions. Gynecological Endocrinology. 2012; 28(10): 815-818, doi: 10.3109/09513590.2012.671387.

10. Xu X, Zheng Q, Zhang Z, et al. Periostin Enhances Migration, Invasion, and Adhesion of Human Endometrial Stromal Cells Through Integrin-Linked Kinase 1/Akt Signaling Pathway. Reproductive Sciences. 2015; 22(9): 1098-1106, doi: 10.1177/1933719115572481.

11. Zheng Qm, Lu Jj, Zhao J, et al. Periostin Facilitates the Epithelial-Mesenchymal Transition of Endometrial Epithelial Cells through ILK-Akt Signaling Pathway. BioMed Research International. 2016; 2016: 1-8, doi: 10.1155/2016/9842619.

12. Logan $P$, Yango P, Tran N. Endometrial Stromal and Epithelial Cells Exhibit Unique Aberrant Molecular Defects in Patients With Endometriosis. Reproductive Sciences. 2017; 25(1): 140-159, doi: 10.1177/1933719117704905.

13. Ganieva U, Nakamura T, Osuka S, et al. Involvement of Transcription Factor 21 in the Pathogenesis of Fibrosis in Endometriosis. The American Journal of Pathology. 2020; 190(1): 145-157, doi: 10.1016/j. ajpath.2019.09.008.

14. Liu L, Li H, Dargahi D, et al. HoxA13 Regulates Phenotype Regionalization of Human Pregnant Myometrium. The Journal of Clinical Endocrinology \& Metabolism. 2015; 100(12): E1512-E1522, doi: 10.1210/jc.2015-2815.

15. Jamaluddin M, Ko YA, Kumar M, et al. Proteomic Profiling of Human Uterine Fibroids Reveals Upregulation of the Extracellular Matrix Protein Periostin. Endocrinology. 2017; 159(2): 1106-1118, doi: 10.1210/en.2017-03018.

16. Chen $X$, Huo L, Ren L, et al. Polycystic Ovary Syndrome is Associated with Elevated Periostin Levels. Experimental and Clinical Endocrinology \& Diabetes. 2018; 127(09): 571-577, doi: 10.1055/a-0752-0061.

17. Ricciardelli C, Lokman NA, Ween MP, et al. WOMEN IN CANCER THEMATIC REVIEW: Ovarian cancer-peritoneal cell interactions promote extracellular matrix processing. Endocrine-Related Cancer. 2016; 23(11): T155-T168, doi: 10.1530/erc-16-0320.

18. Gillan L, Matei D, Fishman DA, et al. Periostin secreted by epithelial ovarian carcinoma is a ligand for alpha(V)beta(3) and alpha(V)beta(5) in- tegrins and promotes cell motility. Cancer Res. 2002; 62(18): 5358-5364, indexed in Pubmed: 12235007.

19. Zhu M, Fejzo M, Anderson $L$, et al. Periostin promotes ovarian cancer angiogenesis and metastasis. Gynecologic Oncology. 2010; 119(2): 337-344, doi: 10.1016/j.ygyno.2010.07.008.

20. Choi K, Yun J, Lee I, et al. Lysophosphatidic acid-induced expression of periostin in stromal cells: Prognoistic relevance of periostin expression in epithelial ovarian cancer. International Journal of Cancer. 2010;128(2): 332-342, doi: 10.1002/ijc.25341.

21. Abbott $K$, Lim JM, Wells $L$, et al. Identification of candidate biomarkers with cancer-specific glycosylation in the tissue and serum of endometrioid ovarian cancer patients by glycoproteomic analysis. PROTEOMICS. 2009; 10(3): 470-481, doi: 10.1002/pmic.200900537.

22. Tian $Y$, Yao Z, Roden R, et al. Identification of glycoproteins associated with different histological subtypes of ovarian tumors using quantitative glycoproteomics. PROTEOMICS. 2011; 11(24): 4677-4687, doi: 10.1002/pmic.201000811.

23. Zhu M, Saxton RE, Ramos L, et al. Neutralizing Monoclonal Antibody to Periostin Inhibits Ovarian Tumor Growth and Metastasis. Molecular Cancer Therapeutics. 2011; 10(8): 1500-1508, doi: 10.1158/1535-7163. mct-11-0046.

24. Karlan B, Dering J, Walsh C, et al. POSTN/TGFBI-associated stromal signature predicts poor prognosis in serous epithelial ovarian cancer. Gynecologic Oncology. 2014; 132(2): 334-342, doi: 10.1016/j.ygyno.2013.12.021.

25. Ryner L, Guan Y, Firestein R, et al. Upregulation of Periostin and Reactive Stroma Is Associated with Primary Chemoresistance and Predicts Clinical Outcomes in Epithelial Ovarian Cancer. Clinical Cancer Research. 2015; 21(13): 2941-2951, doi: 10.1158/1078-0432.ccr-14-3111.

26. Tan T, Yang He, Ye J, et al. CSIOVDB: a microarray gene expression database of epithelial ovarian cancer subtype. Oncotarget. 2015; 6(41): 43843-43852, doi: 10.18632/oncotarget.5983.

27. Sung PL, Jan $\mathrm{YH}$, Lin SC, et al. Periostin in tumor microenvironment is associated with poor prognosis and platinum resistance in epithelial ovarian carcinoma. Oncotarget. 2015; 7(4): 4036-4047, doi: 10.18632/oncotarget.6700.

28. Tang M, Liu B, Bu X, et al. Cross-talk between ovarian cancer cells and macrophages through periostin promotes macrophage recruitment. Cancer Science. 2018; 109(5): 1309-1318, doi: 10.1111/cas.13567.

29. Lu Z, Kamat K, Johnson B, et al. Generation of a Fully Human scFv that binds Tumor-Specific Glycoforms. Scientific Reports. 2019; 9(1), doi: 10.1038/s41598-019-41567-6.

30. Sterzyńska K, Kaźmierczak D, Klejewski A, et al. Expression of Osteoblast-Specific Factor 2 (OSF-2, Periostin) Is Associated with Drug Resistance in Ovarian Cancer Cell Lines. International Journal of Molecular Sciences. 2019; 20(16): 3927, doi: 10.3390/ijms20163927.

31. Kujawa K, Zembala-Nożyńska E, Cortez A, et al. Fibronectin and Periostin as Prognostic Markers in Ovarian Cancer. Cells. 2020; 9(1): 149, doi: 10.3390/cells9010149.

32. Morelli M, Misaggi R, Cello ADi, et al. Tissue expression and serum levels of periostin during pregnancy: a new biomarker of embryo-endometrial cross talk at implantation. European Journal of Obstetrics \& Gynecology and Reproductive Biology. 2014; 175: 140-144, doi: 10.1016/j. ejogrb.2013.12.027.

33. Freis A, Schlegel J, Kuon RJ, et al. Serum periostin levels in early in pregnancy are significantly altered in women with miscarriage. Reproductive Biology and Endocrinology. 2017; 15(1), doi: 10.1186/s12958017-0307-9.

34. Song $\mathrm{Hj}$, Zhang $\mathrm{P}, \mathrm{Guo} \mathrm{Xj}$, et al. The proteomic analysis of human neonatal umbilical cord serum by mass spectrometry. Acta Pharmacologica Sinica. 2009; 30(11): 1550-1558, doi: 10.1038/aps.2009.140.

35. Sasaki H, Roberts J, Lykins D, et al. Novel chemiluminescence assay for serum periostin levels in women with preeclampsia and in normotensive pregnant women. American Journal of Obstetrics and Gynecology. 2002; 186(1): 103-108, doi: 10.1067/mob.2002.118157.

36. Dobreva $M$, Lhoest $L$, Pereira $P$, et al. Periostin as a Biomarker of the Amniotic Membrane. Stem Cells International. 2012; 2012: 1-10, doi: 10.1155/2012/987185.

37. Ivancsó I, Bohács A, Szalay B, et al. Circulating periostin level in asthmatic pregnancy. Journal of Asthma. 2016; 53(9): 900-906, doi: 10.3109/02770903.2016.1165697.

38. Świrska J, Zwolak A, Dudzińska M, et al. Gestational diabetes mellitus - literature review on selected cytokines and hormones of confirmed or possible role in its pathogenesis. Ginekologia Polska. 2018; 89(9): 522-527, doi: 10.5603/gp.a2018.0089. 